\title{
Mito-TEMPO improved L-Arginine- induced acute pancreatitis in rats via TLR-4/ NF-KB/ NLRP3 inflammasome downregulation and antioxidant properties
}

\author{
Hadeel A. Fawzy ${ }^{*}$, Ebtehal M. Fikry. ${ }^{1}$, Hala M. Fawzy ${ }^{1}$, and Asmaa A. Mohammed ${ }^{2}$ \\ ${ }^{1}$ Department of Pharmacology, National Organization for Drug Control and Research, NODCAR, Giza, Egypt. \\ ${ }^{2}$ Department of Pharmacology and Toxicology, Faculty of Pharmacy (Girls), Al-Azhar University, Cairo, Egypt. \\ * Correspondence: e-mail Haymanfawzy@gmail.com Tel : 01142128989 \\ Article history: Received 18-12-2020 Revised 05-01-2021 Accepted 10-01-2021
}

\begin{abstract}
Background: Acute pancreatitis (AP) is a globally significant disease with increasing incidence and prevalence especially in the western world. It has severe complications such as pseudocyst, infection, renal failure, breathing problems, diabetes, malnutrition and chronic pancreatitis. Chronic pancreatitis can lead to pancreatic cancer which is one of the worst types of cancer. Up till now, there is no licensed specific treatment for AP. Objective: This study investigated the therapeutic effects of Mito-TEMPO in the treatment of L-Arginine induced acute pancreatitis in rats besides a possible involved mechanistic pathway. Materials and Methods: Rats randomly allocated into 3 groups: (1) control (received normal saline), (2) L-Arginine treated (300mg/100gm, i.p once) \& (3) L-Arginine+Mito-TEMPO treated (0.7mg/kg/ day, i.p for 7 days). After 7 days from AP induction, serum amylase \& lipase, pancreatic inflammatory mediators "toll-like receptor-4 (TLR-4), nuclear factor kappa-B (NF-кB), NLRP3 inflammasome, caspase-1, interleukin-1 beta (IL-1B)", oxidative parameters "malondialdehyde (MDA), myeloperoxidase (MPO), nitric oxide (NO), reduced glutathione (GSH)", an apoptotic marker "caspase-3" \& pancreatic histopathological changes were estimated for all rats.

Results: L-Arginine induced AP was evidenced by elevation of serum amylase \& lipase, pancreatic inflammatory mediators “TLR-4, NF-кB, NLRP3 inflammasome, caspase-1, IL-1B”, oxidative parameters "MDA, MPO, NO", the apoptotic marker “caspase-3" and infiltration of inflammatory cells proved through hematoxylin \& eosin stain alongside with the reduction of GSH content. All these harmful effects were improved significantly after the administration of Mito-TEMPO. Conclusion: Mito-TEMPO can be introduced as a new therapy for the treatment of acute pancreatitis due to its anti-inflammatory and antioxidant effects.
\end{abstract}

Keywords: L-arginine; acute pancreatitis; Mito-TEMPO; NLRP3 inflammasome; antioxidant; anti-inflammatory

\section{INTRODUCTION}

Acute pancreatitis (AP) is an acute pancreatic inflammatory process that sometimes includes peri-pancreatic tissues and even remote organ systems ${ }^{1}$. It is clinically characterized by abdominal pain and elevated blood pancreatic enzyme levels ${ }^{2}$. The severity of the disease ranges greatly from mild types that affect the pancreas only to significant multi-systemic organ failure disease and death ${ }^{3}$.
$20 \%$ of cases of AP are clinically severe and associated with high morbidity and mortality ${ }^{4}$. AP can lead to pancreatic toxemia that results from the release of the pancreatic broth into the bloodstream which is responsible for impairing cardio-circulatory, pulmonary, renal, and central nervous system functions ${ }^{5}$. Pancreatitis results in more than 800,000 hospital visits in the USA alone and costs more than $\$ 2$ billion per year ${ }^{6}$. 
Noteworthy, Gallstones and alcohol consumption account for more than $70 \%$ of acute pancreatitis ${ }^{7}$. The remaining cases result from the administration of some drugs such as sulfonamides and azathioprine, some infections such as bacteria, viruses or parasites, trauma, hyperlipidemia, hypercalcemia and cigarette smoking ${ }^{8-11}$.

AP differs pathologically from interstitial pancreatitis (Maintained pancreas blood supply), which is generally self-limited, to necrotizing pancreatitis (Interrupted pancreas blood supply), where the level of necrosis can be associated with the severity of the attack and its systemic complications $^{8}$.

Both acute and chronic pancreatitis start by injuries that lead to the pancreas' auto-digestion of its own enzymes ${ }^{12}$. Under normal conditions, many mechanisms protect the pancreas from self-digestion by its secreted enzymes. The acinar cells are responsible for the synthesis, storage, and secretion of digestive zymogens mainly trypsinogen that is activated into trypsin. These zymogens are produced as precursor molecules packaged into granules, and they will not be activated until they are delivered into small intestine ${ }^{9}$. In normal acinar cells, trypsin activity is properly suppressed by pancreatic secretory trypsin inhibitor gene (SPINK1). If trypsin exceeds the (SPINK1) activity owing to excess acinar cell stimulation, acinar cell injury would occur. Alpha-1-antitrypsin (AAT) is a major protease inhibitor in body fluids, which inhibits trypsin by the formation of molar complexes. Numerous reports have associated AAT deficiency with pancreatitis ${ }^{13}$. Pancreatitis occurs when these protective mechanisms are disturbed ${ }^{8}$. These harmful effects will stimulate peri-acinar myofibroblasts and leukocytes to produce pro-inflammatory cytokines and other inflammatory mediators that initiate local inflammation and promote the induction of interstitial edema, necrosis, and apoptosis ${ }^{14}$. They also promote oxidative stress that creates free radicals in acinar cells, resulting in lipid oxidation of the membrane and the activation of transcription factors, including NF-кB, which in turn induce the expression of chemokines that attract mononuclear cells ${ }^{15}$.

Non-invasive methods such as
alcohol-induced,
immune-mediated, diet-induced, gene knockout or
L-arginine or invasive methods such as closed
duodenal loop, antegrade pancreatic duct perfusion,
injection of biliopancreatic duct, a combination of
secretory hyperstimulation with limited exposure to
intraductal bile acid, vascular-induced,

ischemia/reactivity can be used to induce AP in rats 16 .

The AP model induced by L-arginine was first described by Mizunuma et al. ${ }^{17}$ and Tani et al. 18 in rats. As it is inexpensive, non-invasive and simple to induce, this basic amino acid-induced pancreatitis model has become increasingly common because it needs only one or two i.p. Injections for the development of extreme necrotizing disease without any morphological changes in Langerhans islets ${ }^{19,20}$.

There is no complete understanding of the mechanism by which L-arginine causes pancreatitis. Accumulating evidence indicates that in the development of the disease, oxygen-free radicals, nitric oxide (NO) and inflammatory mediators play a key role ${ }^{20}$.

Physicians began looking for an appropriate cure for AP since several decades. After hundreds of clinical trials, no drug therapy is approved for the disease and we have very few successful therapies to date ${ }^{21}$.

Mitochondria are the main organelles that produce adenosine 5-triphosphate (ATP) and reactive oxygen species (ROS) in eukaryotic cells and meanwhile susceptible to oxidative damage. The irreversible oxidative damage in mitochondria has been implicated in various human diseases. Increasing evidence indicates the therapeutic potential of mitochondria-targeted antioxidants (MTAs) for oxidative damage-associated diseases ${ }^{22}$. An increasing number of studies are aimed at developing conventional (nontargeted) antioxidants for restoring physiological conditions during oxidative stress. Although preliminary studies on many cell or animal models showed promising results, the results from clinical trials were sometimes contradictory and caused many side effects ${ }^{23}$. Excessive or inappropriate use of antioxidants may abolish ROS production and result in compensatory upregulation of mitogen-activated protein kinase (MAPK) pathways, which in turn negatively affect the endogenous antioxidant system and normal cell growth and another concern is whether conventional (nontargeted) antioxidants can be absorbed properly and how they are metabolized in different organs. These uncertainties make it difficult to determine the dose of traditional antioxidants used for disease treatment ${ }^{24}$. The most effective way for an antioxidant stepping forward to disease treatment is to conjugate with a carrier, such as lipophilic cations, liposomes, or peptides, to enable its bioactive ingredient to be targeted for transport into the mitochondria. This targeted 
delivery enables antioxidants to achieve high concentration accumulation in cells and mitochondria, thereby protecting cells and tissues from oxidative damage ${ }^{25}$.

The mitochondria-targeted antioxidant, Mito-Tempo (MT), has recently been emerged as one of the most important antioxidants. The antioxidant piperidine nitro-oxide (Tempo) blends this compound with the lipophilic cation triphenylphosphonium (TPP). The tempo is a mimetic superoxide dismutase that in the catalytic cycle dismutases superoxide, while TPP is a membrane-permeant cation that accumulates several hundred folds inside the membrane potential driven mitochondria. This combination produces an effect-targeted mitochondrial chemical ${ }^{26,27}$.

Consequently, this work was aimed to investigate the therapeutic effects of MT on L-arginine induced acute pancreatitis in rats together with studying the inflammatory mechanistic pathway (TLR-4/ NF-кB/ NLRP3 inflammasome) that is activated as a response to increased mitochondrial oxidative stress \& the impact of MT on it.

\section{MATERIALS AND METHODS}

\subsection{Materials}

\subsubsection{Animals}

Adult male Wistar albino rats weighing 160-200 g were used in this study. They were brought from the National Organization for Drug Control and Research animal house (NODCAR, Giza, Egypt). Before testing, the animals were housed in the laboratory room for one week under normal housing conditions, where room temperature was kept at $23-27{ }^{\circ} \mathrm{C}$ and humidity was fixed at $50-70 \%$ with alternating $12 \mathrm{hr}$ luminance and gloom cycles and free entrance to food (standard pellet diet) and water were allowed ad libitum.

Everything in animals' techniques was done according to the Ethics Committee of the faculty of Pharmacy Al-Azhar University, Egypt (permit number: 227/2019). Unnecessary disturbance of animals, pressure and tough maneuver were avoided.

\subsubsection{Chemicals}

L-arginine; (Glaxo Smith Kline) (Egypt),Mito-TEMPO; (Sigma Aldrich) (Germany),Normal saline; (Al-Nasr company) (Egypt), Thio-barbituric acid (Sigma Aldrich)
(Germany), Hydrochloric acid; (Adwic) (Egypt), Tri-chloro acetic acid; (Lobal Chemi) (India.), Potassium dihydrogen orthophosphate; (Adwic)( Egypt), Ortho-dianisidine; (Qualikems) (India.), Hydrogen peroxide(Adwic) (Egypt).

\subsection{Experimental Design}

Rats were allocated in a random way into three groups ( $\mathrm{n}=10$ per each group) as follows: Group 1 (Control) served as normal control; each rat received intra-peritoneal normal saline for 8 days. Group 2 (1-Arginine treated) served as positive control and received a single dose of L-Arginine $(300 \mathrm{mg} / 100 \mathrm{~g}$ body weight in normal saline, intraperitoneal (i.p.) ${ }^{20}$. Group 3 (L-Arginine +Mito-TEMPO treated) received $0.7 \mathrm{mg} / \mathrm{kg}$ body weight/ day, i.p. for 7 days started 24 hours after L-arginine injection ${ }^{27,28}$.

All rats were decapitated on the $9^{\text {th }}$ day of the experiment. Animals were decapitated under light anesthesia at the end of the experiment, and the pancreas was isolated from each rat, and then the used animals were frozen till being incinerated. A part of the pancreas was homogenized in $10 \% \mathrm{w} / \mathrm{v}$ saline ${ }^{29}$, centrifuged at $4000 \mathrm{rpm} 4^{\circ} \mathrm{C}$ for $15 \mathrm{~min}$ and then the supernatants were frozen at $-80^{\circ} \mathrm{C}$ for further evaluation of oxidative stress biomarkers, inflammatory mediators and apoptosis via caspase- 3 activity. Another part was handled for the recognition and determination of the quantity of NLRP3 inflammasome protein by western blot analysis. A part from the pancreas (3 rats / group) was preserved in $10 \%$ formalin-saline and treated for histopathological analysis using hematoxylin and eosin (H\&E) stain for the detection of inflammatory scores and the examination was done blind to the histologist.

\subsubsection{Evaluated parameters in the serum}

\subsection{1.a. Serum amylase and lipase}

The serum content of alpha-amylase and lipase were accomplished according to the manufacturer's instructions using reagent kits (spectrum diagnostics company, Cairo, Egypt) 30,31 . The principle of amylase determination is that alpha amylase catalyzes the hydrolysis of 2-chloro-4-nitrophenyl1-galactopyranosyl-maltosid e (GALG2-CNP) to glucose polymers and p-nitrophenyl oligosaccaride at short chain producing 2-chloro4-nitrophenol (CNP). The increased extinction can be measured by spectrophotometry at $405 \mathrm{~nm}$ and results are proportional to the activity of alpha amylase present 
in the sample. The principle of lipase determination is that the synthetic substrate (DGMRE) is split by Lipase to yield the colored final product Methylresorufin. The increasing absorbance of the red Methylresorufin is measured photo-metrically.

\subsection{1.b. Serum glucose}

The serum glucose content was accomplished according to the manufacturer's instructions using a reagent kit (Biodiagnostic Company, Giza, Egypt) 32. The principle of glucose determination depends on that glucose reacts with the first reagent to give hydrogen peroxide and then hydrogen peroxide reacts with the second reagent to give the Colored quinonemine that can be measured photo-metrically.

\subsubsection{Biochemical measurements in the} pancreatic homogenate

\subsection{2.a. Assessment of oxidative stress biomarkers}

The pancreatic content of reduced glutathione (GSH) and nitrite/nitrate $\left(\mathrm{NO}_{\mathrm{x}}\right)$ were accomplished according to the manufacturer's instructions using a reagent kit (Biodiagnostic Company, Giza, Egypt) ${ }^{33}$, 34. The method of GSH detection is based on the reduction of 5,5 dithiobis (2 - nitrobenzoic acid) ( DTNB ) with glutathione (GSH) to produce a yellow compound. The reduced chromogen directly proportional to GSH concentration and its absorbance can be measured at $405 \mathrm{~nm}$ and the method of $\left(\mathrm{NO}_{\mathrm{x}}\right)$ depends on that in acid medium and in the presence of nitrite the formed nitrous acid diazotise sulphanilamide and the product is coupled with N-(1-naphthyl) ethylenediamine. The resulting azo dye has a bright reddish - purple color which can be measured at $540 \mathrm{~nm}$.

On the other hand, myeloperoxidase (MPO) activity and malondialdehyde content (MDA) were determined biochemically according to Bonyadi, M., and Buege, J.A. ${ }^{35,36}$, respectively. MDA was determined biochemically where thiobarbituric acid reacts with MDA in acidic medium at $95^{\circ} \mathrm{C}$ for 30 min to produce a pink colored product; its absorbance is measured at $534 \mathrm{~nm}$ using a single beam spectrophotometer. The principle of MPO depends of that MPO reacts with the substrate to give a color that can be measured photo-metrically.

\subsection{2.b. Assessment of Inflammatory biomarkers}

The pancreatic content was tested for toll-like receptor-4 (TLR-4), caspase-1, interleukin-1 beta
(IL-1 $\beta$ ) and nuclear factor- kappa B (NF-кB) were accomplished according to the manufacturer's instructions using reagent rat ELISA kits; (Cusabio; China, lifespan biosciences; the USA, Bioassay technology laboratory; UK and Mybiosource; the USA, respectively). The assay employs the quantitative sandwich enzyme immunoassay technique. Antibody specific for any of the antigens has been pre-coated onto a microplate. Standards and samples are pipetted into the wells and any antigen present is bound by the immobilized antibody. After removing any unbound substances, a biotin-conjugated antibody specific for the antigen is added to the wells. After washing, avidin conjugated Horseradish Peroxidase (HRP) is added to the wells. Following a wash to remove any unbound avidin-enzyme reagent, a substrate solution is added to the wells and color develops in proportion to the amount of the antigen bound in the initial step. The color development is stopped, and the intensity of the color is measured.

\subsection{2.c. Assessment of apoptosis via caspase-3} activity

Caspase- 3 activity was determined according to the manufacturer's instructions using a reagent rat ELISA kit (Bioassay technology laboratory, UK). The assay employs the quantitative sandwich enzyme immunoassay technique. Antibody specific for caspase-3 has been pre-coated onto a microplate. Standards and samples are pipetted into the wells and any caspase- 3 present is bound by the immobilized antibody. After removing any unbound substances, a biotin-conjugated antibody specific for caspase- 3 is added to the wells. After washing, avidin conjugated Horseradish Peroxidase (HRP) is added to the wells. Following a wash to remove any unbound avidin-enzyme reagent, a substrate solution is added to the wells and color develops in proportion to the amount of caspase- 3 bound in the initial step. The color development is stopped, and the intensity of the color is measured.

\subsection{2.d. Assessment of total protein}

Total proteins were accomplished according to the manufacturer's instructions using a reagent kit (Biodiagnostic Company, Giza, Egypt) ${ }^{37}$. The method depends on that in the presence of an alkaline cupric sulfate; the protein produces a violet color, the intensity of which is proportional to their concentration.

\subsection{2.e. Quantitative determination of NLRP3} inflammasome by western blot analysis 
Using RIPA Lysis and Extraction Buffer, total proteins were collected from pancreatic tissue (Bio BASIC INC. Marhham Ontario L3R 8T4 Canada). According to development directions, BIO BASIC INC. Markham Ontario L3R 8T4 Canada calculated the protein concentration. Equal protein amounts (20 $\mu \mathrm{g}$ per lane) were loaded onto the gel and separated by 10 percent (w/v) acrylamide SDS-polyacrylamide gels, followed by electrophoresis and blotting on to Polyvinylidene fluoride membranes (EMD Millipore). The membranes were blocked at room temperature for $1 \mathrm{hr}$ in tris-buffered saline with Tween 20 (TBST) buffer and 3 percent bovine serum albumin (BSA) and co-incubated overnight with TEST-diluted anti-NLRP3 inflammasome primary antibodies (Thermofisher, USA) in a shaker at $4^{\circ} \mathrm{C}$. After rinsing with TBST, the membranes were co-incubated for $2 \mathrm{~h}$ on a shaker at room temperature with HRP-conjugated secondary antibody (Goat anti-rabbit IgG- HRP-lmg Goat mab -Novus Biologicals). The membranes were treated with $5 \mathrm{ml}$ of BCIP/NBT solution (Invitrogen; Thermo Fisher Scientific, Inc.) following additional rinsing with TBST, as directed by the manufacturer. To evaluate the protein ladders, Quantity One 1-D analysis software version 4.6.3 (Bio-Rad Laboratories, Inc.) was used. Expression levels of proteins have been normalized to those of the housekeeping gene $(\beta \text {-actin })^{38}$.

\subsection{Statistical Analysis}

Data were stated as means \pm SEM. Comparisons among means were done using One-Way ANOVA then Tukey multiple comparisons test. Statistical analysis was accomplished using Graph Pad Prism software (version 5); a probability level of less than 0.05 was believed to be statistically significant.

\section{RESULTS}

\subsection{Effect of mito-TEMPO on serum amylase} and lipase

Administration of L-arginine (figure 1A and B) caused a remarkable significant increase in both serum amylase and lipase by 81 and $73 \%$, respectively as compared to the control group. On the other hand, administration of mito-TEMPO reduced the serum amylase significantly by $54 \%$ and the serum lipase significantly by $47 \%$ as compared to the L-arginine group.

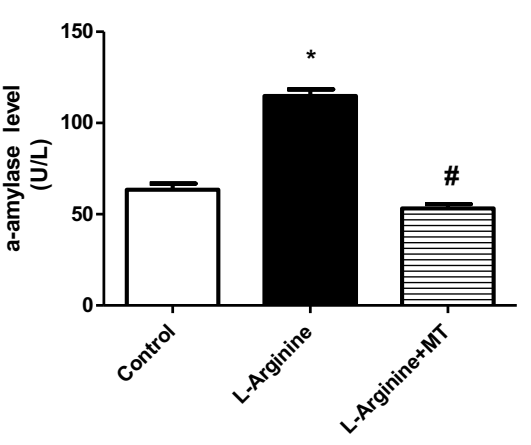

(A)

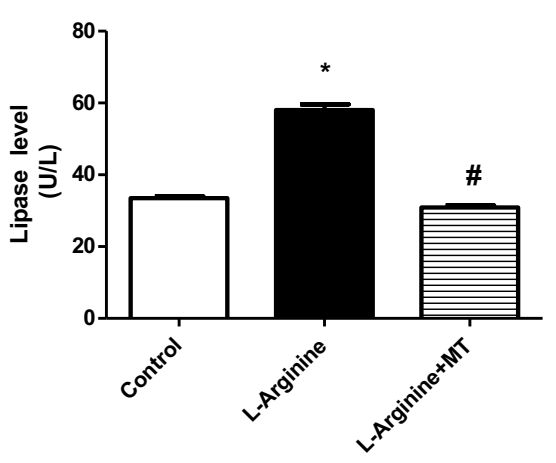

(B)

Figure 1: The influence of mito-TEMPO $(0.7 \mathrm{mg} / \mathrm{kg}$, i.p for 7 days) on serum amylase (A) and lipase (B) in $\mathrm{L}$-arginine induced acute pancreatitis rats (L-arginine, $300 \mathrm{mg} / 100 \mathrm{gm}$, i.p once). Data are expressed as the mean values \pm S.E.M. $(n=6)$. Data were analyzed by one-way ANOVA followed by Tukey-Kramer multiple comparisons test. Significance * $(p<0.05)$ versus control, \# $(\mathrm{p}<0.05)$ versus L-arginine groups. MT; mito-TEMPO.

\subsection{Effect of mito-TEMPO on serum glucose}

There is no significant difference among the three groups in the serum glucose level. This is clarified in the table (1).

\subsection{Effect of mito-TEMPO on oxidative stress biomarkers}

L-arginine decreased GSH content markedly by $62 \%$ as compared to the control group, however, administration of mito-TEMPO raised the GSH content significantly to 3 folds as compared to the L-arginine group. This is shown in figure (2A). Moreover, L-arginine injection caused a notable significant rise in $\mathrm{NO}_{\mathrm{x}}$ content which was $39 \%$ as compared to the control group, while, administration of mito-TEMPO diminished the $\mathrm{NO}_{x}$ content significantly by $40 \%$ as compared to the L-arginine 
group. This is illustrated in the table (2). In addition, injection of L-arginine produced a high increment in MDA content that was $48 \%$ as compared to the control group, however, administration of mito-TEMPO caused a prominent decline in MDA content that was $33 \%$ as compared to the 1 -arginine group. This is seen in figure (2B). Also, administration of L-arginine augmented the MPO activity to 8 folds as compared to the control group, while administration of mito-TEMPO caused a significant drop in MPO by $87 \%$ as compared to the $\mathrm{L}$-arginine group. This is exemplified in the table (2).

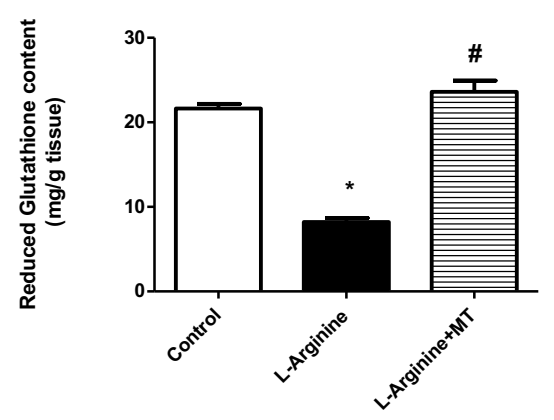

(A)

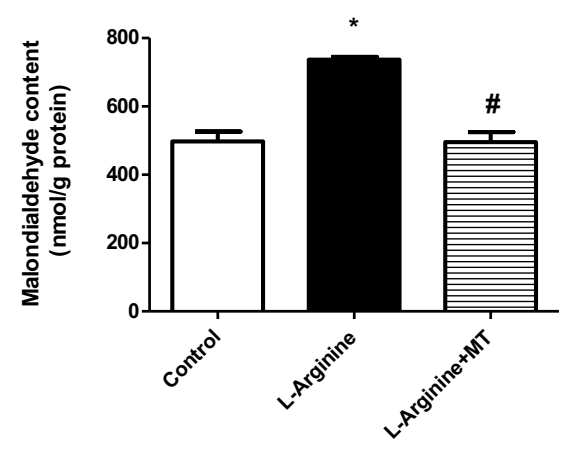

(B)

Figure 2: The influence of mito-TEMPO $(0.7 \mathrm{mg} / \mathrm{kg}$, i.p for 7 days) on pancreatic reduced glutathione (A), malondialdehyde contents (B) in L-arginine induced acute pancreatitis rats (L-arginine, $300 \mathrm{mg} / 100 \mathrm{gm}$, i.p once). Data are expressed as the mean values \pm S.E.M. $(n=6)$. Data were analyzed by one-way ANOVA followed by Tukey-Kramer multiple comparisons test. Significance * $(p$ $<0.05)$ versus control, \# $(\mathrm{p}<0.05)$ versus L-arginine groups. MT; mito-TEMPO.
Table 1: The influence of mito-TEMPO $(0.7 \mathrm{mg} / \mathrm{kg}$, i.p for 7 days) on serum glucose and total protein in L-arginine induced acute pancreatitis rats (L-arginine, $300 \mathrm{mg} / 100 \mathrm{gm}$, i.p once). Data are expressed as the mean values \pm S.E.M. $(n=6)$. Data were analyzed by one-way ANOVA followed by Tukey-Kramer multiple comparisons test. Significance * $(\mathrm{p}<0.05)$ versus control, \# $(\mathrm{p}<0.05)$ versus L-arginine groups. MT; mito-TEMPO.

\begin{tabular}{lll}
\hline Groups & $\begin{array}{l}\text { Glucose } \\
(\mathrm{mg} / \mathrm{dl})\end{array}$ & $\begin{array}{l}\text { Total protein } \\
(\mathrm{g} / \mathrm{ml})\end{array}$ \\
\hline Control & $165.7 \pm 14.38$ & $0.02777 \pm 0.002192$ \\
L-arginine & $160.2 \pm 11.28$ & $0.02575 \pm 0.000112$ \\
L-arginine+MT & $165.1 \pm 010.96$ & $0.0293 \pm 0.002218$ \\
\hline
\end{tabular}

\subsection{Effect of mito-TEMPO on inflammatory biomarkers}

Injection of L-arginine caused a significant elevation in TLR- 4 content to 4 folds as compared to the control group. In contrast, administration of mito-TEMPO shrank the TLR-4 content significantly by $52 \%$ as compared to the L-arginine group. This is illustrated in figure (3A). Furthermore, administration of L-arginine amplified the NF- $\mathrm{KB}$ content significantly by $87 \%$ as compared to the control group. On the other hand, administration of mito-TEMPO lessened the NF-кB content significantly by $21 \%$ compared with the L-arginine group. These results are demonstrated in figure (3B). Also, Intra-peritoneal injection of L-arginine caused a major significant rise in NLRP3 inflammasome content to 6 folds as compared to the control group. Noteworthy, mito-TEMPO administration declined the NLRP3 inflammasome content significantly by $58 \%$ as compared to the L-arginine group. This is shown in figure (3C). Moreover, administration of L-arginine produced a huge significant escalation in caspase- 1 activity to 6 folds compared to the control group. Conversely, mito-TEMPO induced a remarkable significant improvement by decreasing caspase- 1 activity by $62 \%$ as compared to the L-arginine group. These results are explained in figure (3D). Additionally, L-arginine brought a significant intensification in IL- $1 \beta$ content by $30 \%$ as compared to the control group. In contrast, mito-TEMPO diminished IL- $1 \beta$ content significantly by $49 \%$ as compared to the L-arginine group. These results are illustrated in the table (2). 

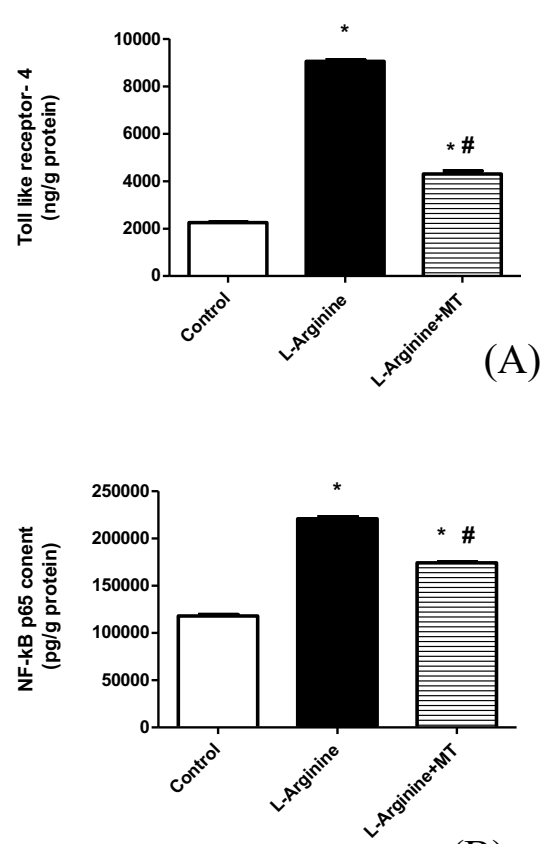

(B)

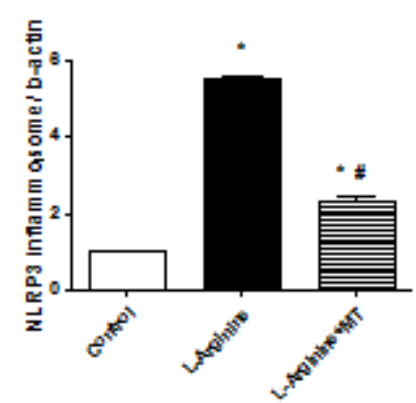

(C)
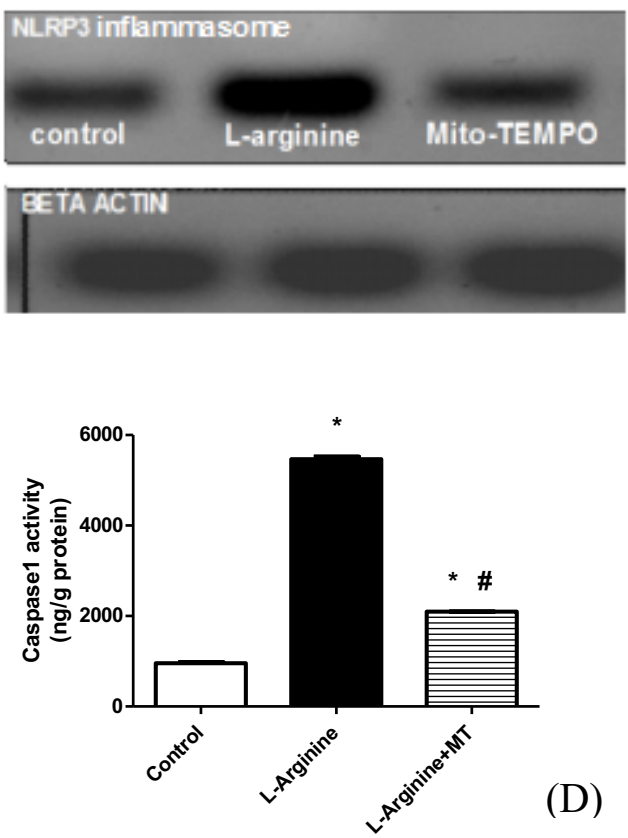

(D)

Figure 3: The influence of mito-TEMPO $(0.7 \mathrm{mg} / \mathrm{kg}$, i.p for 7 days) on pancreatic Toll like receptor-4 (A), NF-кВ (B), NLRP3 inflammasome contents (C) and caspase-1 activity (D) in L-arginine induced acute pancreatitis rats (L-arginine, $300 \mathrm{mg} / 100 \mathrm{gm}$, i.p once). Data are expressed as the mean values \pm S.E.M. $(n=6)$. Data were analyzed by one-way ANOVA followed by Tukey-Kramer multiple comparisons test. Significance * $(p$ $<0.05)$ versus control, \# $(\mathrm{p}<0.05)$ versus L-arginine groups. MT; mito-TEMPO.

Table 2: The influence of mito-TEMPO $(0.7 \mathrm{mg} / \mathrm{kg}$, i.p for 7 days) on pancreatic MPO, $\mathrm{NO}_{\mathrm{x}}$ and IL-1 $\beta$ contents in L-arginine induced acute pancreatitis rats (L-arginine, 300mg/100gm, i.p once). Data are expressed as the mean values \pm S.E.M. $(n=6)$. Data were analyzed by one-way ANOVA followed by Tukey-Kramer multiple comparisons test. Significance * $(p<0.05)$ versus control, \# $(\mathrm{p}<0.05)$ versus L-arginine groups. MT; mito-TEMPO.

\begin{tabular}{llll}
\hline Groups & $\begin{array}{l}\text { MPO } \\
(\mathrm{u} / \text { g protein })\end{array}$ & $\begin{array}{l}\text { NO } \\
(\mu \mathrm{mol} / \mathrm{g} \\
\text { protein })\end{array}$ & $\begin{array}{l}\text { IL-1 } \beta \\
(\mathrm{pg} / \mathrm{g} \text { protein })\end{array}$ \\
\hline & $0.2534 \pm$ & $2.558 \pm$ & \\
Control & 0.02181 & 0.07148 & $10595 \pm 34.69$ \\
L-arginine & $1.927 \pm$ & $3.552 \pm$ & $13728 \pm 99.38^{*}$ \\
L-arginine+MT & $0.1844^{*}$ & $0.1625^{*}$ & $7068 \pm 0.8735^{* \#}$ \\
& $0.2566 \pm$ & $2.135 \pm$ & \\
& $0.0008859^{\#}$ & $0.1659^{\#}$ & \\
\hline
\end{tabular}

\subsection{Effect of mito-TEMPO on Total protein}

There is no significant difference among the three groups in the total protein content. This is clarified in the table (1).

\subsection{Effect of mito-TEMPO on caspase-3 activity (apoptotic biomarker)}

L-arginine injection raised the caspase- 3 activity by $75 \%$ as compared to the control group. Instead, mito-TEMPO administration attenuated the caspase- 3 activity significantly by $50 \%$ as compared to the L-arginine group. This is elucidated in figure (4). 


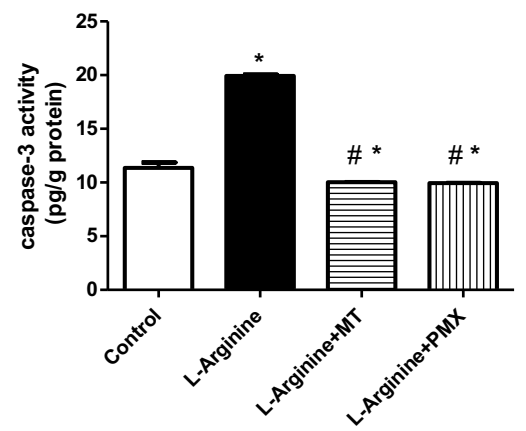

Figure 4: The influence of mito-TEMPO $(0.7 \mathrm{mg} / \mathrm{kg}$, i.p for 7 days) on pancreatic caspase- 3 activity in L-arginine induced acute pancreatitis rats (L-arginine, 300mg / $100 \mathrm{gm}$, i.p once). Data are expressed as the mean values \pm S.E.M. $(n=6)$. Data were analyzed by one-way ANOVA followed by Tukey-Kramer multiple comparisons test. Significance * $(\mathrm{p}<0.05)$ versus control, ${ }^{\#}(\mathrm{p}<0.05)$ versus L-arginine groups. MT; mito-TEMPO.

\subsection{Estimation of pancreatic histopathology examination using Hematoxylin and Eosin stain}

Pancreatic tissues were examined using $\mathrm{H} \& \mathrm{E}$ stain.

The control group (figure (5A)) showed normal pancreatic acini which were closely packed and consisted of a single layer of pyramidal cells converging into a narrow central lumen .cytoplasm showed intense basal basophilia and apical acidophilia. The pancreatic duct was normal. Islets of Langerhans appeared as compact spherical or egg-shaped masses embedded within the acinar exocrine tissue with its differentiated cells.

Administration of L-arginine (figure (5 B)) triggered many pathological alternations where pancreatic acini were absent in all pancreatic lobules. Severe edema and inflammatory cell aggregations in the interstitial space were seen. Also, the pancreatic duct displays mild inspissation with severe proliferation in all lobules. Also, pancreatic islets showed intact cells with moderate to severe vacuolations among its elements.

Mito-TEMPO (figure (5C)) elicited moderate improvement in the form of reformation of scattered accumulation of pancreatic acini in all pancreatic lobules. Moderate to severe inflammatory cell infiltration is still observed in the interstitial space with severely congested blood vessels and perivascular edema. The pancreatic islet displays congested blood capillaries and the vacuolation among its element still present. The proliferated pancreatic duct was still seen with mild peri-ductal fatty cells infiltration.

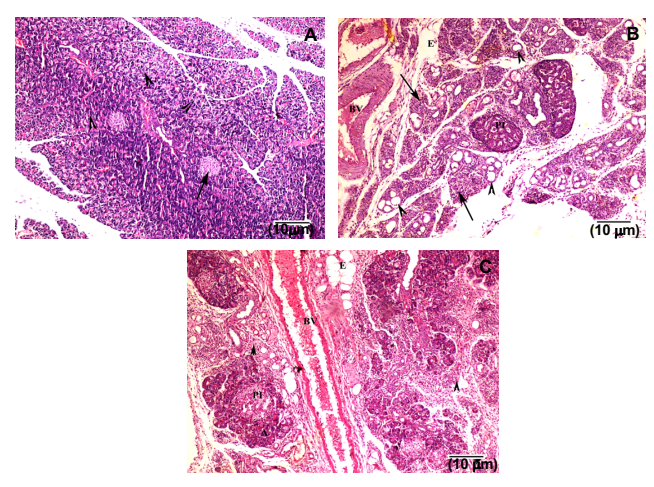

Figure 5: Representative $\mathrm{H}$ and $\mathrm{E}$ staining images to detect the influence of mito-TEMPO $(0.7 \mathrm{mg} / \mathrm{kg}$, i.p for 7 days $)$ on pancreatic histopathological examination in L-arginine induced acute pancreatitis rats (L-arginine, 300mg / $100 \mathrm{gm}$, i.p once), where (A) is a photomicrograph of a section of the pancreas of the control group, (B) is a photomicrograph of a section of the pancreas of the L-arginine group and $(\mathrm{C})$ is a photomicrograph of a section of the pancreas of the "L-arginine + mito-TEMPO" group.

\section{DISCUSSION}

This study supports the anti-inflammatory and the antioxidant activities of mito-TEMPO in the treatment of L-arginine- induced acute pancreatitis in rats. The $\mathrm{L}$-arginine model of pancreatitis has many advantages over other models because it is very good for investigating the early and late phases of pancreatitis according to the dose and it is also suitable for researching extra-pancreatic organ damage and its mechanisms ${ }^{16}$. Mito-TEMPO is a well-known mitochondria-specific superoxide scavenger that can accumulate in mitochondria preventing the generation of oxidative stress and alleviating the adverse effects of the conventional antioxidants such as the required dose and compensatory upregulation of MAPK pathways, which in turn negatively affect the endogenous antioxidant system and normal cell growth ${ }^{24,39}$.

In the present study, L-arginine initiated acute pancreatitis shown by a high level of serum amylase and lipase through stimulation of oxidative stress revealed by the high increment of MPO activity, MDA content and $\mathrm{NO}_{\mathrm{x}}$ content, as well as severe decline in reduced GSH content. These results agree with a previous study of L-arginine- induced acute pancreatitis in a rat model ${ }^{40}$. 
Moreover, L-arginine induced acute pancreatitis in rats ${ }^{54}$. This may be due to the use of only a pancreatitis via elevation of inflammatory single dose of L-arginine that induced milder acute biomarkers such as TLR-4 content, NF-KB content, pancreatitis than the one induced by repeated doses, so NLRP3 inflammasome content, caspase-1activity causing injury in the $\beta$ cells and affecting the endocrine and IL- $1 \beta$ content. These results are in harmony with function.

previous studies of acute pancreatitis in mice and rat models ${ }^{41-44}$.

Furthermore, L-arginine triggered apoptosis via high caspase- 3 activity, and this is following a previous study of acute pancreatitis in rats ${ }^{45}$.

Noteworthy, administration of mito-TEMPO alleviated the oxidative stress that was revealed by the significant reduction in MPO activity, MDA content and $\mathrm{NO}_{\mathrm{x}}$ content as well as replenishment of reduced GSH content. These results are following a previous study where mito-TEMPO diminished MDA content and elevated reduced GSH significantly in neuropathic pain in rats ${ }^{46}$; however, these results are opposite to another study where mito-TEMPO failed to affect NO content and MPO level in a sepsis model in mice ${ }^{47}$.

Mito-TEMPO alleviated oxidative stress as it is a mitochondria-targeted antioxidant and is a SOD mimetic that dismutates superoxide anion or radical in the catalytic cycle ${ }^{26}$.

Moreover, in our study mito-TEMPO exerted anti-inflammatory effects that were revealed by a remarkable decline in TLR-4, NF-кB, NLRP3 inflammasome and IL-1 $\beta$ contents as well as caspase-1 activity. This is in harmony with a previous study where mito-TEMPO inhibited NLRP3 pathway activation in a model of renal damage in mice ${ }^{48}$.

It is supposed that the mechanism by which Mito-TEMPO produced anti-inflammatory effects maybe by the prevention of mitochondrial reactive oxygen species that were involved in activation of TLR-4 and hence NF-кB signaling and this will lead to prevention of NLRP3 inflammasome activation and hence caspase- 1 and its related cytokines like IL-1 $\beta$ and IL-1 $8^{49-51}$.

In addition, mito-TEMPO exerted anti-apoptotic activity that was clarified by a significant reduction in caspase- 3 activity and this is in harmony with a previous study in a model of diabetic cardiomyopathy in rats ${ }^{52}$. It is supposed that the anti-apoptotic effect of mito-TEMPO is due to the up-regulation of Bcl-2 and its antioxidant activities ${ }^{52}$. Besides, mito-TEMPO also prevented the activation of the mitochondrial pathway of apoptosis and mitochondrial oxidative stress-mediated pro-inflammatory signaling through NF- $\mathrm{B}^{53}$.

In our study, the blood glucose level wasn't affected in all groups and this is opposite to another study of acute

In our study, L-arginine caused pancreatic edema, degenerative changes of pancreatic acini and inflammatory cell infiltration shown by H\&E stain of the pancreas and these results are in accordance with a previous study in acute pancreatitis in rats ${ }^{55}$.

On the other hand, mito-TEMPO elicited a moderate improvement in pancreatic histology revealed by $\mathrm{H} \& \mathrm{E}$ stain. These results are in harmony with a previous study, where mito-TMPO showed alleviation of hepatotoxicity of acetaminophen on the liver in mice ${ }^{56}$. These results may be due to the anti-inflammatory and antioxidant effects of mito-TEMPO.

The authors recommend further studies to assess the utilization of mito-TEMPO in the treatment of acute pancreatitis in humans.

\section{CONCLUSION}

Our results indicated that mito-TEMPO played an important role in attenuating L-arginine induced acute pancreatitis in vivo; possibly by inhibiting mitochondrial reactive oxygen species (mtROS) production and NLRP3 inflammasome activation, therefore targeting mtROS may be an effective strategy in ameliorating acute pancreatitis.

\section{Acknowledgment}

The authors are grateful to Dr. Shrouk Mohamed Ahmed for her supporting role in the analysis of histopathology and Dr. Laila Rashed for her help in the western blot analysis of NLRP3 inflammasome.

\section{Conflict of interest}

The authors declare no conflict of interest.

\section{Ethical approval statement}

Everything in animals' techniques was done according to the Ethics Committee of the faculty of Pharmacy Al-Azhar University, Egypt (permit number: 227/2019). Unnecessary disturbance of animals, pressure and tough

maneuver was avoided.

\section{Authors' contributions}


All authors were hand by hand in each part of this research.

\section{Funding statement}

This research hasn't received any fund from anywhere.

\section{REFERENCES}

1. Working GI, APA APG. IAP/APA evidence-based guidelines for the management of acute pancreatitis. Pancreatology 2013;13:e1.

2. Nwafo NA. Acute pancreatitis following oesophagogastroduodenoscopy. Case Reports. 2017;2017.

3. Pandol SJ, Saluja AK, Imrie CW, Banks PA. Acute pancreatitis: bench to the bedside. Gastroenterology. 2007;133(3):1056. e1-. e25.

4. Chatila AT, Bilal M, Guturu P. Evaluation and management of acute pancreatitis. World J Clin Cases. 2019;7(9):1006.

5. Bassi C, Falconi M, Butturini G, Pederzoli P. Early complications of severe acute pancreatitis. Surgical Treatment: Evidence-Based and Problem-Oriented: Zuckschwerdt; 2001.

6. Pendharkar SA, Mathew J, Zhao J, Windsor JA, Exeter DJ, Petrov MS. Ethnic and geographic variations in the incidence of pancreatitis and post-pancreatitis diabetes mellitus in New Zealand: a nationwide population-based study. NZ Med J. 2017;130(1450):55-68.

7. Herreros-Villanueva M, Hijona E, Bañales JM, Cosme A, Bujanda L. Alcohol consumption on pancreatic diseases. WJG. 2013;19(5):638.

8. Antonius DW. Acute PancreatitisEtiology, Pathogenesis, Pathophysiology and The Current Trend in Its Management and Prevention. The Indonesian Journal of Gastroenterology, Hepatology, and Digestive Endoscopy. 2019;20(1):27.

9. Wang G-J, Gao C-F, Wei D, Wang C, Ding S-Q. Acute pancreatitis: etiology and common pathogenesis. WJG. 2009;15(12):1427.

10. Rawla P, Bandaru SS, Vellipuram AR. Review of infectious etiology of acute pancreatitis. Gastroenterology Research. 2017;10(3):153.

11. Edderkaoui M, Thrower E. Smoking and pancreatic disease. JCT. 2013;4(10A):34.
12. Lin K, Gao F, Chen Q, Liu Q, Chen S. Framework for interpretation of trypsinantitrypsin imbalance and genetic heterogeneity in pancreatitis. SJG. 2015;21(4):198.

13. Kavutharapu S, Nagalla B, Abbagani V, Porika SK, Akka J, Nallari P, et al. Role of proteases and antiprotease in the etiology of chronic pancreatitis. SJG. 2012;18(6):364.

14. Manohar M, Verma AK, Venkateshaiah SU, Sanders NL, Mishra A. Pathogenic mechanisms of pancreatitis. WJGPT. 2017;8(1):10.

15. Yu JH, Kim H. Oxidative stress and inflammatory signaling in cerulein pancreatitis. WJG. 2014;20(46): 17324.

16. $\mathrm{Su} \mathrm{KH}$, Cuthbertson $\mathrm{C}$, Christophi C. Review of experimental animal models of acute pancreatitis. HPB. 2006;8(4):264-86.

17. Mizunuma $\mathrm{T}$, Kawamura S, Kishino $\mathrm{Y}$. Effects of injecting excess arginine on rat pancreas. JN. 1984;114(3):467-71.

18. Tani S, Itoh H, Okabayashi Y, Nakamura T, Fujii M, Fujisawa T, et al. New model of acute necrotizing pancreatitis induced by excessive doses of arginine in rats. Digestive diseases and sciences. 1990;35(3):367-74.

19. Kui B, Balla Z, Vasas B, Végh ET, Pallagi $\mathrm{P}$, Kormányos ES, et al. New insights into the methodology of L-arginine-induced acute pancreatitis. PLOS. 2015;10(2):e0117588.

20. Hegyi P, Rakonczay Jr Z, Sári R, Góg C, Lonovics J, Takács $\mathrm{T}$, et al. L-arginine-induced experimental pancreatitis. WJG. 2004;10(14):2003.

21. Afghani E, Pandol SJ, Shimosegawa T, Sutton R, Wu BU, Vege SS, et al. Acute pancreatitis-progress and challenges: a report on an international symposium. Pancreas. 2015;44(8):1195.

22. Cadenas E, Davies KJ. Mitochondrial free radical generation, oxidative stress, and aging. Free radical biology and medicine. 2000;29(3-4):222-30.

23. Salehi B, Martorell M, Arbiser JL, Sureda A, Martins N, Maurya PK, et al. Antioxidants: positive or negative actors? BIOMHC. 2018;8(4):124.

24. Cerimele F, Battle T, Lynch R, Frank DA, Murad E, Cohen C, et al. Reactive oxygen signaling and MAPK activation distinguish Epstein-Barr Virus (EBV)-positive versus EBV-negative 
Burkitt's lymphoma. PNAS. 2005;102(1):175-9.

25. Jiang Q, Yin J, Chen J, Ma X, Wu M, Liu $\mathrm{G}$, et al. Mitochondria-Targeted Antioxidants: A Step towards Disease Treatment. Oxidative med. cell. longev. 2020;2020.

26. Du K, Farhood A, Jaeschke H. Mitochondria-targeted antioxidant Mito-Tempo protects against acetaminophen hepatotoxicity. ARCH TOXICOL. 2017;91(2):761-73.

27. Li C, Sun H, Xu G, McCarter KD, Li J, Mayhan WG. Mito-Tempo prevents nicotine-induced exacerbation of ischemic brain damage. J Appl Physiol. 2018;125(1):49-57.

28. Reddy S, Jacob T, Garg P, Sahni P. Effect of dexamethasone and infliximab on the severity of L-arginine induced murine acute pancreatitis. HPB. 2018;20:S209.

29. Lu G, Tong Z, Ding Y, Liu J, Pan Y, Gao $\mathrm{L}$, et al. Aspirin protects against acinar cells necrosis in severe acute pancreatitis in mice. BIOMED RES INT. 2016;2016.

30. Winn-Deen ES, David H, Sigler G, Chavez R. Development of a direct assay for alpha-amylase. Clinical chemistry. 1988;34(10):2005-8.

31. Lorentz K. Lipase. Clinical laboratory diagnostics 1st ed Frankfurt: TH-Books Verlagsgesellschaft. 1998:95-7.

32. Trinder P. Determination of glucose in blood using glucose oxidase with an alternative oxygen acceptor. ACB. 1969;6(1):24-7.

33. Beutler E, Duron O, Kelly M. Glutathione reagent and method-patent. J Lab Clin Med. 1963;61:882.

34. Montgomery H, Dymock JF. Determination of nitrite in water. RSC . 1961. p. 414-\&.

35. Bonyadi M, Waldman SD, Liu D, Aubin JE, Grynpas MD, Stanford WL. Mesenchymal progenitor self-renewal deficiency leads to age-dependent osteoporosis in Sca-1/Ly-6A null mice. PNAS. 2003;100(10):5840-5.

36. Buege JA, Aust SD. [30] Microsomal lipid peroxidation. METHOD ENZYMOL. 52: Elsevier; 1978. p. 302-10.

37. Gornall AG, Bardawill CJ, David MM. Determination of serum proteins by means of the biuret reaction. J. Biol. Chem. 1949;177(2):751-66.

38. Zhu J, Wu S, Hu S, Li H, Li M, Geng X, et al. NLRP3 inflammasome expression in peripheral blood monocytes of coronary heart disease patients and its modulation by rosuvastatin. MOI. 2019;20(2):1826-36.

39. Yang S-G, Park H-J, Kim J-W, Jung J-M, Kim M-J, Jegal H-G, et al. Mito-TEMPO improves development competence by reducing superoxide in preimplantation porcine embryos. Sci. Rep. 2018;8(1):1-10.

40. Abdel-Aziz AM, Rifaai RA, Abdel-Gaber SA. Possible mechanisms mediating the protective effect of cilostazol in L-arginine induced acute pancreatitis in rats: role of cGMP, cAMP, and HO-1. Naunyn Schmiedebergs Arch Pharmacol. 2020:1-12.

41. Li Y, Pan Y, Gao L, Zhang J, Xie X, Tong $\mathrm{Z}$, et al. Naringenin protects against acute pancreatitis in two experimental models in mice by NLRP3 and Nrf2/HO-1 pathways. MEDIAT INFLAMM. 2018;2018.

42. Siriviriyakul P, Chingchit T, Klaikeaw N, Chayanupatkul M, Werawatganon D. Effects of curcumin on oxidative stress, inflammation and apoptosis in L-arginine induced acute pancreatitis in mice. Heliyon. 2019;5(8):e02222.

43. Sharif R, Dawra R, Wasiluk K, Phillips P, Dudeja V, Kurt-Jones E, et al. Impact of toll-like receptor 4 on the severity of acute pancreatitis and pancreatitis-associated lung injury in mice. Gut. 2009;58(6):813-9.

44. Rau B, Paszkowski A, Lillich S, Baumgart $\mathrm{K}$, Möller $\mathrm{P}$, Beger HG. Differential Effects of Caspase-1/Interleukin-1 $\beta$ Converting Enzyme on Acinar Cell Necrosis and Apoptosis in Severe Acute Experimental Pancreatitis. Lab Invest. 2001;81(7):1001-13.

45. Shafik NM, Abou-Fard GM. Ameliorative effects of curcumin on Fibrinogen-Like protein-2 gene expression, some OxidoInflammatory and apoptotic markers in a rat model of 1-Arginine-Induced acute pancreatitis. J. Biochem. Mol. Toxicol. 2016;30(6):302-8.

46. Zhan L, Li R, Sun Y, Dou M, Yang W, He $\mathrm{S}$, et al. Effect of mito-TEMPO, a mitochondria-targeted antioxidant, in rats with neuropathic pain. NERPEZ. 2018;29(15):1275-81.

47. Rademann P, Weidinger A, Drechsler S, Meszaros A, Zipperle J, Jafarmadar M, et al. Mitochondria-targeted antioxidants SkQ1 and MitoTEMPO failed to exert a long-term beneficial effect in murine 
polymicrobial sepsis. Oxid. Med. Cell. Longev. 2017;2017.

48. Wen Y, Liu Y, Tang T, Lv L, Liu H, Ma $\mathrm{K}$, et al. NLRP3 inflammasome activation is involved in Ang II-induced kidney damage via mitochondrial dysfunction. Oncotarget. 2016;7(34):54290.

49. Ding W, Guo H, Xu C, Wang B, Zhang M, Ding F. Mitochondrial reactive oxygen species-mediated NLRP3 inflammasome activation contributes to aldosterone-induced renal tubular cells injury. Oncotarget. 2016;7(14):17479.

50. Nisr RB, Shah DS, Ganley IG, Hundal HS. Proinflammatory NFkB signalling promotes mitochondrial dysfunction in skeletal muscle in response to cellular fuel overloading. Cell. Mol. Life Sci. 2019; 76(24):4887-904.

51. Ferrero-Andrés A, Panisello-Roselló A, Roselló-Catafau J, Folch-Puy E. NLRP3 Inflammasome-Mediated Inflammation in Acute Pancreatitis. Int. J. Mol. Sci. 2020;21(15):5386.

52. Ni R, Cao T, Xiong S, Ma J, Fan G-C, Lacefield JC, et al. Therapeutic inhibition of mitochondrial reactive oxygen species with mito-TEMPO reduces diabetic cardiomyopathy. Free Radic. Biol. Med. 2016;90:12-23.

53. Mazumder S, De R, Sarkar S, Siddiqui AA, Saha SJ, Banerjee C, et al. Selective scavenging of intra-mitochondrial superoxide corrects diclofenac-induced mitochondrial dysfunction and gastric injury: a novel gastroprotective mechanism independent of gastric acid suppression. Biochem. Pharmacol. 2016;121:33-51.

54. Abd El-Rahman HS. Antioxidant activity and Anti-Inflammatory Effects of Lemon grass on L-Arginine-Induced Acute Pancreatitis in Rats Middle East J. Appl. Sci.2019; 1177-1189

55. Soliman ME-s, Kefafy MA, Mansour MA, Ali AF, Esa WAII. Histological study on the possible protective effect of pentoxifylline on pancreatic acini of 1-arginine-induced acute pancreatitis in adult male albino rats. Menoufia Med. J. 2014;27(4):801.

56. Abdullah-Al-Shoeb M, Sasaki K, Kikutani S, Namba N, Ueno K, Kondo Y, et al. The Late-Stage Protective Effect of Mito-TEMPO against Acetaminophen-Induced Hepatotoxicity in Mouse and Three-Dimensional Cell
Culture

Models. 